\title{
EL DETERMINISMO EN LA TEORÍA CONTIN- GENTE: ORÍGENES DE SUS FUNDAMENTOS TEÓRICOS PARA SU COMPRENSIÓN
}

Determinism in the contingent theory: origins of its foundations theorists to understand

\section{Gerardo Zapata Rotundo*}

Resumen: Este artículo de naturaleza teórica, tiene como propósito analizar una de las teorías que ha tenido mayor impacto en el estudio y comprensión del desarrollo, diseño y cambio de la organización, como lo es la teoría contingente, cuyos inicios se remontan a la década del 60 del siglo xx. Esta teoría ha sido utilizada por una gran cantidad de investigaciones como enfoque teórico de referencia, partiendo de la primacía de que no existe una mejor forma de organización, sino que la más apropiada depende de la naturaleza de factores contextuales internos y externos y de su ajuste con las variables de la organización, situación que, a su vez, determinará su desempeño y resultados. Se considera entonces a los factores contingentes como una fuente directa del cambio organizacional y de la forma organizativa que se adopte. De este modo, para lograr el objetivo de este trabajo se aborda estudio de su origen, características y sus implicaciones $\mathrm{y}$, finalmente, se propone un modelo teórico denominado: Modelo determinista-contingente de la organización.

Especialista en Contaduría; Profesor-Investigador Titular del Decanato de Administración y Contaduría de la Universidad Centroccidental "Lisandro Alvarado", Venezuela. E-Mail: zapager@yahoo.com 
Palabras claves: Ajuste organizacional, entorno, estructura organizativa, diseño organizativo, factores contingente, teoría contingente.

Abstract: This theoretical paper, we aim to analyze one of the theories that has had the greatest impact on the study and understanding of the development, design and organizational change, such as the contingent theory whose beginnings can be traced back to the early 60 s of last century. This theory has been used by a lot of research and theoretical reference approach, starting from the premise that there is no better form of organization, but the most appropriate depends on the nature of internal and external contextual factors and their adjustment with the variables of the organization, a situation that, in turn, determine its performance and results. The contingent factors as a direct source of organizational change and organizational form adopted is then considered. Thus, to achieve the objective of this paper study of their origin, characteristics and their implications is discussed, and finally called a theoretical model is proposed: DeterministicContingent Model of the organization.

Keywords: Organizational Fit, Environmental, Organizational Structure, Organization Design, Contingent Factors, Contingency Theory. 
El determinismo en la teoría contingente: Orígenes de sus fundamentos teóricos para su comprensión

\section{Introducción}

En el marco de la teoría de la organización, se han desarrollado diferentes perspectivas teóricas que explican el diseño y comportamiento de las organizaciones (Zapata y Mirabal; 2013). Así, durante las primeras décadas del siglo veinte la dirección de la empresa y los teóricos de la organización tuvieron la tendencia a ignorar el entorno y aplicaron principios universalistas para planificar, organizar, coordinar y controlar las organizaciones, constituyéndose, por tanto, en sistemas cerrados. La idea la expresa de manera acertada Morgan (1990: 23) cuando dice:

Los teóricos clásicos de la dirección y de la dirección científica fueron pioneros y ofrecieron a los directivos la mejor manera de organizar. Los pioneros teóricos creyeron que habian descubierto los principios de la organización, los cuales, si se seguian, resolverían, más o menos, los problemas de la dirección para siempre. Ahora, sólo tenemos que observar una organización contemporánea para ver que estaban completamente equivocados en su visión.

A partir de los años sesenta, fueron evolucionando distintos modelos cada vez más elaborados que representaban el enlace entre un conjunto de factores condicionantes de la estructura y de los procesos de la organización (Burns y Stalker, 1961'; Lawrence y Lorsch, 1967a, 1967b; Thompson, 1967; Donaldson, 1996, 2001), modelos que dieron forma a lo que se conoce hoy como la teoría contingente de la organización.

En numerosas investigaciones, se argumenta que la organización responde en forma predecible a las condiciones que impone el contexto donde participa, ajustando sus objetivos a esas demandas. De ahí, la diferencia entre lo que postulaban los primeros

\footnotetext{
1 Burns y Stalker (1961) se consideran, en buena parte, los iniciadores de la teoría contingente que quedó definida en los trabajos pioneros de Lawrence y Lorsch (1967a, 1967b).
} 
investigadores o teóricos de la administración con los del enfoque contingente, quienes han tratado la estructura y los procesos, y muchos aspectos de la organización, como variables dependientes de un grupo de factores contextuales externos e internos a ella.

Así, el enfoque contingente parte de la idea de que no existe una manera única de organización, y en su forma más pura se corresponde con un modelo determinista puesto que toma la visión de que esos factores contextuales, o de contingencia, determinan la naturaleza de los diseños de sus procesos y estructura organizativa, con lo cual es necesario el " $f i t$ " o ajuste de las variables organizacionales con esos condicionantes para que pueda alcanzar un desempeño superior o al menos poder sobrevivir (Donaldson, 2001; Child et al., 2003; Sillince, 2005; Simon, 2007; Pertusa, et al., 2010; Zapata y Mirabal; 2013). Se corresponde entones a un enfoque teórico bajo una perspectiva de adaptación de la organización.

De esta manera, este trabajo tiene como propósito abordar el análisis de una de las teorías que ha tenido una mayor influencia e impacto en el estudio y comprensión del desarrollo, diseño y cambio de la organización. En tal sentido, para cumplir con su propósito se han desarrollado tres apartados adicionales a esta introducción. En el primero, se abordan los aspectos relacionados con la definición, formación y características de la teoría contingente, pasando por el estudio de los trabajos clásicos y seminales que dieron origen a sus postulados y que permitieron consolidarla como teoría. El segundo apartado, se presenta una variante de esta perspectiva tal como lo es la teoría contingente estructural. Y en un tercer apartado, se analiza el significado del fit, o ajuste, dentro del marco de la teoría contingente. Finalmente, se ofrece en el último apartado, y a modo conclusión, una propuesta de modelo teórico determinista-contingente de la organización. 


\section{La teoría contingente y su acción determinista}

Postula la proposición de que uno o más elementos contextuales (internos o externos a la organización) operan como determinantes principales del diseño de la organización y de sus procesos organizativos, fundamentada en tres principios básicos: (1) no hay una forma óptima de organizar la empresa; (2) no existen maneras de organizar igual de eficientes; y (3) el entorno es considerado como dado, lo que quiere decir que la organización tiene pocas posibilidades de influir o controlar su situación frente a él, lo que significa que los criterios para evaluar el desempeño de la organización son definidos principalmente desde ese entorno (Lawrence and Lorsch, 1967a, 1967b; Galbraith, 1973; Donaldson, 1996, 2001; Buttermann, et al.,2008; Zapata et al, 2009; Tenhiälä, 2011; Volberda, 2012). Planteándose una circunstancia en la cual tiene que adaptarse para para sobrevivir, es decir; se presenta como un sistema abierto-adaptativo a las condiciones de su contexto.

La orientación determinista del enfoque contingente no se centra en el individuo, sino en las condiciones externas dentro de las cuales se desenvuelve y desarrolla el funcionamiento de la organización, observándose el comportamiento de sus miembros como un producto de las reacción y exigencias establecidas por esas condiciones contextuales (Pfeffer y Salancik, 1978; Astley y Van de Ven, 1983; Donaldson, 2001; Rond y Thietart, 2007, Carvalho, 2011; Volberda, 2012). Por lo que el determinismo está asociado a una relación causa-efecto, en la cual quienes dirigen a la organización actúan dentro de las restricciones y limitaciones que imponen los factores de contingencia, principalmente el entorno.

En este sentido, la perspectiva contingente argumenta que la organización depende e impulsa la identificación y descripción de las variables externas e internas de las cuales depende el comportamiento, el diseño y el desempeño de la organización (Miles y Snow, 1978, Donaldson, 2001; Wong et al., 2011; Volberda, 2012), por lo que "a partir del reconocimiento de la inexistencia de modelos 
estructurales óptimos, señala que el diseño de organizaciones eficientes depende de la adecuación de las variables internas -O de diseño- al estado y evolución de las variables externas -o de contingencias"- (De la Fuente et al., 2002:262), referido esto a la hipótesis de congruencia ${ }^{2}$. Lo cual significa que la organización que alcance la mejor correspondencia o ajuste con los factores del entorno, será la más eficiente y tendrá, en consecuencia, una mayor probabilidad de supervivencia.

Es de destacar algunos trabajos pioneros desarrollados durante las décadas de los años 60 y 70 del siglo pasado, los cuales establecieron las bases conceptuales de la teoría contingente e identificaron un conjunto de factores contingentes.

Burns y Stalker (1961). La idea fundamental desarrollada por estos autores en su trabajo de 20 empresas de Inglaterra y Escocia, es que las formas organizativas y los procesos directivos están relacionados directamente con ciertos elementos del entorno, principalmente con los cambios tecnológicos y del mercado. De esta manera, su grado de estabilidad determinará los sistemas de control y supervisión de las actividades, la manera cómo fluirá la información, y la forma como las decisiones y las acciones deberán ser tomadas dentro de la organización. Los autores manifiestan que "las evidencias empíricas ofrecidas nos llevan a considerar al sistema de dirección como una variable dependiente (...) con respecto a los requerimientos en los factores extrínsecos. Esos factores extrínsecos son, de acuerdo a nuestro punto de vista, identificables como los distintos índices de cambios tecnológicos y de mercado" (Burns y Stalker, 1961:96). Se observa en alguna medida en el pensamiento de esos autores, la idea de la acción determinista del entorno.

2 De la Fuente et al. (2002: 262) señalan que la hipótesis de congruencia "nos sitúa ante el auténtico problema del analista organizativo, a saber: conocer el contexto organizativo y ajustar las variables de diseño a las circunstancias ambientales particulares de cada organización, en cada momento”. 
En su trabajo, sugieren y caracterizan dos formas organizativas: las mecánicas -centralizadas, con alta formalización y especializaciónadaptadas a condiciones estables del entorno; y las orgánicas -descentralizadas, con baja formalización y especializaciónadaptadas a entornos cambiantes, inestables y con alta incertidumbre. Al conjunto de esos postulados lo denominan: teoría orgánica de la organización.

Es de destacar que el determinismo subyacente a los planteamientos de Burns y Stalker, fundamentalmente cuando hablamos de formas orgánicas, no se corresponde a un modelo en el que los componentes internos de la organización dan una respuesta inmediata o automática a los condicionantes del entorno, como ocurre en las formas mecánicas o burocráticas, donde el ajuste puede darse, en cierta forma, de manera predecible; es decir, un determinismo clásico explicado a través de un proceso de ajuste entre, por ejemplo, la tecnología, el tamaño y la estructura organizativa necesaria para que la organización sea eficiente. Esto es, en parte, debido al hecho de que una organización es orgánica puesto que dentro de ella hay objetivos compartidos, márgenes de libertad para actuar, opinar, participar, decidir y poner la iniciativa al servicio de la innovación y de esa manera dar respuesta a los nuevos retos impuestos por el entorno, dándosele espacio importante a la perspectiva voluntarista. Existe así una amplia vinculación entre los cambios del entorno, acompañado con incertidumbre y la necesidad de los individuos de crear y gestionar nuevos conocimientos e innovar. Como resultado se produce un ajuste o determinismo algo distinto, más complejo y menos predecible, donde el entorno, caracterizado por los cambios y la innovación, obliga a la empresa a ser más orgánica pero sin que sepamos exactamente cómo será esa respuesta orgánica.

Grupo de Aston. El programa del grupo de Aston durante varios años se dedicó a estudiar una serie de variables o dimensiones de diseño organizativo (Pugh et al. 1968), el contexto de la estructura de la organización (Pugh et al., 1969b) y una taxonomía de las 
estructuras burocráticas (Pugh et al., 1969a). Sus estudios empíricos los basaron en una muestra de empresas de Inglaterra. Siendo estas investigaciones, los fundamentos seminales para una gran cantidad de estudios desarrollados posteriormente dentro del marco de la teoría de la organización, y de manera muy especial en el diseño organizativo.

Así, los objetivos del programa de Aston fueron:

1) Encontrar de qué manera una organización estructura sus actividades;

2) Ver si es posible, o no, crear métodos fiables de validación estadística para medir las diferencias estructurales entre las organizaciones; $\mathrm{y}$

3) Examinar qué restricciones impone el contexto de la organización sobre la estructura organizativa (Pugh, 1973).

En el desarrollo de sus investigaciones definieron siete variables contextuales consideradas independientes, y cinco variables estructurales dependientes. Las variables contextuales identificadas fueron:

(a) Origen e historia de la empresa, referida a la fundación de la empresa, cambios de propietarios, localización, entre otros;

(b) Propiedad y control, referida al tipo de propiedad -pública o privada-, y a la concentración de esa propiedad y su control en pocas o muchas manos;

(c) Tamaño de la organización, según el número de empleados, activos netos y posición en el mercado;

(d) La cantidad de bienes y servicios producidos por la organización;

(e) Tecnología, la definen como la secuencia de técnicas físicas utilizadas en el flujo de trabajo, o el grado de integración alcanzado en los procesos de trabajo de la organización; 
(f) Localización, se refiere al número de centros operativos de la organización dispersos geográficamente;

(g) Interdependencia, refleja la relación y grado de dependencia de la organización con su entorno social, tales como clientes, proveedores, competidores, sindicatos y organizaciones gubernamentales y políticas (Pugh et al., 1969b; Pugh y Hickson, 1976).

Por otra parte en la definición de las variables estructurales encontraron que la especialización ${ }^{3}$, la estandarización y la formalización ${ }^{4}$ estaban significativamente relacionadas con lo cual las agruparon en un sólo factor que lo denominaron estructuración de actividades (Pugh et al., 1968; Pugh y Hickson, 1976; Pugh, 1981). Sin embargo, no encontraron una relación muy estrecha y claramente definida entre la estructuración de actividades y la variable centralización -denominada concentración de autoridad-, lo que parecía una variable con un comportamiento independiente.

La falta de una relación clara entre la centralización y la estructuración de actividades llevó al grupo de Aston a concluir que no existía un tipo ideal de burocracia tal como lo había planteado Weber, sino a considerar distintos tipos de burocracia con diferentes grados de centralización -de ahí, en parte, su clasificación de diferentes burocracias-. Lo anterior lo manifiestan cuando dicen: "esto parece (refiriéndose a sus resultados) ${ }^{5}$ refutar la hipótesis ofrecida por la tradición Weberiana de burocracia ideal, y de la idea de que en la burocracia las decisiones pasan siempre a los niveles superiores, y que la formalización y centralización están

3 La especialización, responde a la descomposición del trabajo en múltiples tareas asignadas de manera únicas a cada puesto de trabajo o persona. Lo que conduce a trabajos limitados y con cualificaciones únicas.

4 La formalización, constituye el grado en el cual las reglas, procedimientos, políticas, planes, instrucciones, tareas, funciones y comunicaciones están escritos, y sirven para controlar y regular el comportamiento de los miembros de la organización.

5 Paréntesis nuestro. 
positivamente relacionadas" (Pugh et al., 1968:82). La última variable la denominan configuración, la cual puede ser medida por varios índices: ámbito de control, cadena de mando y el crecimiento vertical (Pugh et al., 1968; Pugh, 1973; Pugh, 1981).

En general, el grupo de Aston señala que los resultados demuestran que las variables contextuales tienen un papel sobresaliente en la determinación de la estructura organizativa y en las funciones de la organización, lo que supone pensar en la presencia de una relación causal, donde la dirección tiene un papel secundario en la determinación de dicha estructura, limitándose su actuación a gestionar los procedimientos o mecanismos necesarios para que la organización alcance el nivel de ajuste necesario con los factores condicionantes del entorno. Con esto se observa una visión determinista dentro de la perspectiva contingente.

Lawrence y Lorsch (1967a, 1967b). La contribución principal de estos autores se centra en la idea de establecer cuál es la estructura más adecuada para una organización compleja en un entorno determinado, siendo las más eficaces aquellas que poseen las mejor capacidad de ajustar sus variables organizativas con las demandas del entorno. En ese sentido, manifiestan que "(...) hemos intentado sacar a la luz las posibilidades de un nuevo enfoque basado en la investigación al que, provisionalmente, le damos el nombre de teoría contingente de la organización" (Lawrence y Lorsch, (1967a: 202-203), denominación que finalmente fue definitiva.

Lawrence y Lorsch (1967a, 1967b) definen la estructura de la organización en términos del grado de diferenciación e integración. Ese grado variará en función a las exigencias particulares del entorno, proponiendo que una de las razones principales de la diferenciación en distintos subsistemas es que la organización pueda manejar con eficiencia diferentes sub-entornos -mercado, producción, económico-. Como resultado de ello, diferentes unidades organizativas se corresponden a diferentes sub-entornos, $\mathrm{y}$ cada unidad tendrá su propia estructura para adaptarse y responder 
a él. Por ejemplo, el sub-entorno del departamento de mercadeo es muy diferente al de producción o investigación, y por consiguiente su estructura organizativa.

En sus trabajos Lawrence y Lorsch (1967a, 1967b) tratan a la diferenciación como el estado de segmentación de la organización, cada uno de los cuales tiende a desarrollar características particulares y estructuras diferentes en relación con los condicionantes del entorno. Se incluyen en esa segmentación las diferencias existentes entre las distintas unidades orgánicas en cuanto a la orientación de los objetivos, la formalización de sus estructuras, los tipos de relaciones interpersonales, la orientación de las actividades respecto al tiempo -proceso de planificación-, y las habilidades y conocimientos necesarios para llevar a cabo las tareas (Lawrence y Lorsch, 1967a). Además, nos dicen los autores, cuando las organizaciones emprenden tareas más complejas tienden a complicarse internamente, surgen, por tanto, nuevas unidades organizativas necesarias para atender las diferentes partes especializadas del entorno, reflejándose en el hecho de que "cualquier grupo de gerentes o directores tienen un área limitada de control. Cada uno tiene la capacidad de tratar sólo con una parte del entorno total" (Lawrence y Lorsch, 1967a:17). Conocido esto también como el principio de ámbito de control.

En todo caso, lo fundamental en Lawrence y Lorsch es que la complejidad que implica más dinamismo y cambio, y mayor dificultad de la tarea debido a una mayor complejidad del sector donde se encuentra la empresa, lleva a la necesidad de diferenciar:

Más complejidad del sector y de las tareas conduce a más diferenciación para poder hacer frente a los diferentes aspectos de la complejidad, y a causa de ello a una mayor necesidad de integración y/ o coordinación para conseguir unidad de propósito entre las unidades diferenciadas, $y$ de esa forma garantizar el cumplimento de los objetivos de la organización. 
Entre sus conclusiones, Lawrence y Lorsch manifiestan que los grados de diferenciación e integración de las organizaciones eficaces dependen de los atributos y exigencias específicas de cada entorno; por ello, cuanto más diferenciada está una organización más difícil resultará resolver distintos puntos de vista y establecer una eficaz colaboración entre las partes; es decir, más difícil resulta encontrar una integración eficaz. Si su estado de diferenciación es alto, entonces la organización necesitará mecanismos de integración -coordinaciónmucho más complejos que aseguren un nivel adecuado de cohesión y conexión entre los diferentes subsistemas o partes de la organización. En otras palabras, mecanismos capaces de resolver los problemas derivados de la complejidad relacional. ${ }^{6}$

De esta manera Lawrence y Lorsch nos indican que en un entorno más diverso y dinámico, las organizaciones han de estar altamente diferenciadas e integradas, siendo más difícil solucionar los distintos puntos de vista y llevar a cabo una eficaz colaboración entre las diferentes unidades organizativas. Por otra parte, en un entorno más estable y menos diversificado, la organización ha de estar menos diferenciada y es más sencillo llegar a un alto grado de integración. En resumen, para diferentes entornos deben haber diferentes niveles de diferenciación e integración y, por tanto, diferentes repuestas organizativas.

Blau (1970). En su trabajo seminal sobre la relación del tamaño, como factor de contingencia interno, y la estructura organizativa plantea que el incremento del tamaño de la organización produce un aumento en su diferenciación traducido en: (a) un crecimiento horizontal -número de divisiones, departamentos y secciones-; y (b) un crecimiento vertical -número de niveles en la jerarquía-; situaciones que generan problemas de coordinación. ${ }^{7}$ Igualmente

6 La complejidad relacional se asocia: (a) con el número de componentes en el sentido de diferenciación vertical, horizontal y espacial presentes en cualquier organización; y (b) con la intensidad de las interacciones, la interdependencia de trabajos, la capacidad de integración y coordinación entre las partes constitutivas, y los problemas que surgen de esa coordinación (Zapata y Caldera, 2008).

7 El tamaño de la organización medido por el número de empleados. 
El determinismo en la teoría contingente: Orígenes de sus fundamentos teóricos para su comprensión

indica que el índice de crecimiento de la diferenciación va decreciendo con el aumento del tamaño de la organización -una relación curvilínea-. Asimismo, sugiere que el aumento del tamaño produce:

1. Un aumento de la subdivisión de responsabilidades que facilita la supervisión.

2. Una ampliación del ámbito de control.

3. Una reducción de la intensidad administrativa que ocasiona economías de escala en la supervisión; o sea, hay una relación negativa entre el tamaño y la intensidad administrativa. ${ }^{8}$

4. El aumento de la diferenciación, por el aumento del tamaño, producirá: una mayor heterogeneidad entre las unidades y una mayor homogeneidad dentro de ellas, y un aumento en su grado de formalización.

Child (1973). Su trabajo se dirigió fundamentalmente a examinar las variables contingente que consideraba pronosticadoras de la estructura de la organización. Tomó para su análisis una muestra de ochenta y dos empresas Británicas, seleccionadas de acuerdo a seis sectores de la industria y por el número de empleados. Algunas de sus conclusiones fueron:

a) La especialización, la formalización, la estandarización, la expansión vertical y la descentralización -variables estructuralesse incrementan con el aumento del tamaño de la organización. Las grandes organizaciones tienden a asignar un mayor número de empleados a roles especializados, a crear un amplio rango

\footnotetext{
${ }^{8} \mathrm{La}$ intensidad administrativa se corresponde al número de empleados que ocupan cargos de gerentes, asesores administrativos y profesionales en relación a la cantidad de personal ubicado en la base de la pirámide organizacional directamente vinculados con las actividades operativas (Astley, 1985; McKinley, 1987; Donaldson, 2001), constituyendo, por tanto, una medida del porcentaje o proporción entre empleados, supervisores o profesionales y el resto de trabajadores de la organización (Zapata y Hernández, 2011).
} 
de unidades orgánicas, a tener una mayor estandarización de los procedimientos y documentos, y a delegar las decisiones en los niveles inferiores de la estructura organizativa. Pero encontró, al igual que Blau (1970), que el efecto del tamaño sobre las variables estructurales declina con su aumento.

De acuerdo a la idea anterior, la relación entre el tamaño y las variables estructurales es una representación curvilínea. En la figura n. 1 se observa que al aumentar de izquierda a derecha la variable $\mathbf{X}$-números de empleados- también lo hace $\mathbf{Y}$-variables estructurales-, pero a partir de un cierto valor de $\mathbf{X}$, la variable $\mathbf{Y}$ comienza a disminuir y por tanto la curva tiende a declinar.

\section{Figura N. ${ }^{\circ} 1$}

El tamaño de la empresa y las variables estructurales

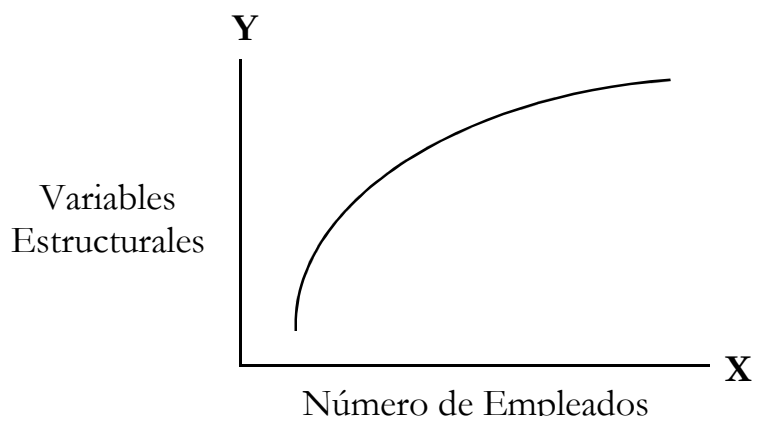

b) La descentralización está acompañada por un incremento en los procedimientos estándares y el uso de documentos -formalización- para mantener el control en la organización. Asimismo, indica que las organizaciones con un gran número de empleados con baja cualificación, usualmente utilizan mecanismos de control poco complejos tal como la supervisión directa. Por otra parte, también señala Child (1973), que el aumento en la complejidad de las tareas hace posible pensar en la implementación de controles por medio de mecanismos 
indirectos o impersonales, por ejemplo la formalización. Los niveles de complejidad, recalca el autor, están influenciados por la automatización e integración tecnológica, la cantidad de sitios de operación y los contactos a través de las fronteras de la organización. Por lo tanto, el control burocrático y la formalización son variables dependientes del nivel de complejidad.

c) El grado de burocratización es más evidente y extensivo en grandes organizaciones; es decir, está fuertemente determinado por el tamaño. En palabras de Donaldson (2001), significa que el aumento del tamaño lleva a la estructuración de grandes burocracias -aumento de la especialización, las reglas, los procedimientos escritos y el establecimiento de estándares de trabajo-. Esto es así, puesto que en la medida que la organización va creciendo se establece un distanciamiento cada vez mayor entre el centro principal de poder y el resto de la organización, por consiguiente los gerentes y supervisores buscan apoyarse en reglas, documentos y procedimientos que estandaricen las actividades y proporcionen guías de conducta que faciliten el establecimiento de mecanismos de supervisión y control indirecto sobre una mayor cantidad de empleados.

Galbraith $\left(\mathbf{1 9 7 3}^{9}, 1977\right)$. Ofrece un modelo contingente el cual concibe a la organización como una red de procesamiento de información basado en dos ideas esenciales:

1) La incertidumbre del entorno puede afectar las tareas o el trabajo realizado en la organización; así "mientras más incertidumbre hay sobre las tareas, mayor será la cantidad de información que deberá ser procesada por los tomadores de decisiones durante la ejecución de sus tareas para poder alcanzar un nivel adecuado de actuación" (Galbraith, 1977: 36). De esta forma, sostiene que si las tareas son entendidas antes de que se lleven a cabo, entonces pueden ser programadas con anticipación y desarrolladas con

\footnotetext{
${ }^{9}$ Las citas y referencias corresponden a la versión en castellano de 1977.
} 
facilidad; y cuando es al contrario, durante la realización de las tareas se irá adquiriendo los conocimientos, las habilidades y los recursos necesarios para ejecutarlas. En otras palabras, un procesamiento emergente de información.

2) En función a la incertidumbre de las tareas, la organización empleará distintas estrategias de procesamiento de información. Galbraith (1973, 1977) propone dos: (a) reducir la necesidad de procesamiento de información a través de la creación o consumo de recursos holgados -por ejemplo, aumentando el tiempo programado de producción, las horas hombres invertidas y los plazos de entrega de los productos; y (b) aumentar la capacidad de procesamiento de información por medio de la inversión en sistemas de información vertical -tecnología de información, programas de almacenamiento de información, formalización de los canales de comunicación, etc.- o la creación de modos de comunicación lateral y procesos de toma de decisión conjunta -contacto directo, roles de enlace, grupos de enlace, rol integrador, gerente integrador y diseño en forma de matriz-. Ambas estrategias podrán aplicarse simultáneamente en una misma organización.

Pfeffer y Salancik (1978). Bajo el marco de la teoría de dependencia de recursos, sugieren un modelo que explica la relación causal entorno-organización a través de la influencia de los factores de contingencia sobre la distribución del poder y la sustitución de los gerentes. Ese modelo lo representan en la figura N. ${ }^{\circ}$.

Se observa:

1) El entorno con sus contingencias e incertidumbre influye en la distribución del poder y el control dentro de la organización, proveyendo más poder a algunas unidades que a otras;

2) La distribución del poder y el control afecta la permanencia y selección de los gerentes; 
Figura N. ${ }^{\circ} 2$

Relación Entorno-Organización: Distribución de poder y control

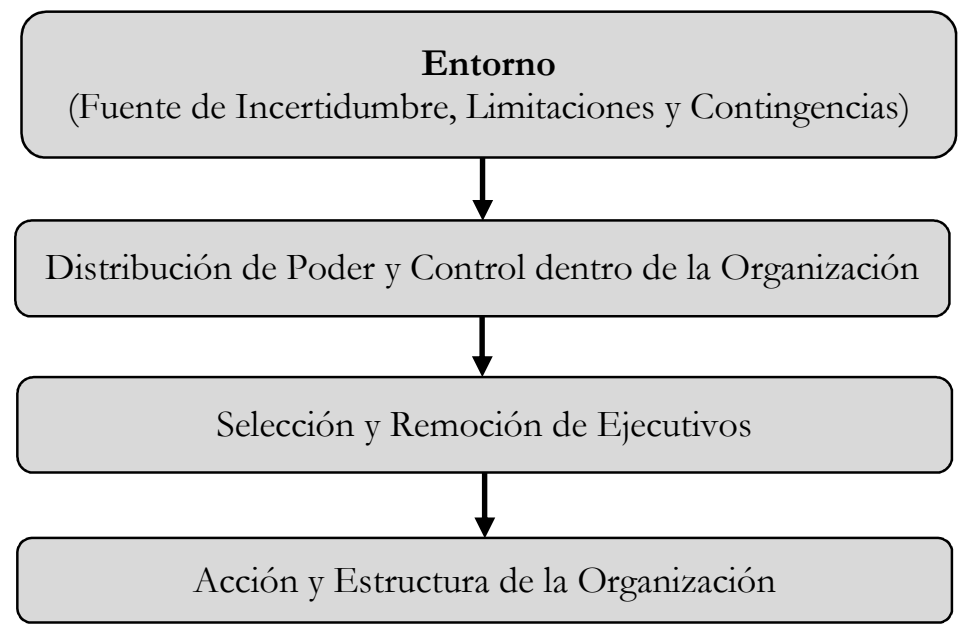

Fuente: Pfeffer y Salancik (1978: 229)

3) Las políticas de la organización y su estructura son el resultado de las decisiones que han sido afectadas por la distribución del poder y el control; $y$

4) Los administradores que mantienen el control de la organización, tienen cierta influencia sobre las actividades y la estructura organizativa (Pfeffer y Salancik, 1978: 228-229).

En suma, el modelo de Pfeffer y Salancik representa una relación de influencias del entorno sobre la organización, donde se observa un impacto directo del entorno sobre la distribución del poder y el control, y de éstos, a su vez, como variables moderadoras en la definición de la acción y la estructura organizativa -efecto indirecto de los factores de contingencia-. No obstante se observa que este enfoque reconoce, en cierta forma, el papel de la dirección en las decisiones estructurales, cuestión que deja de lado el enfoque clásico contingente.

Finalmente, Moreno-Luzón et al. (2001:122) sostienen: "La aportación del enfoque contingente consiste en mostrar mediante 
sólidos trabajos empíricos que las diferentes circunstancias condicionantes de la empresa -o factores de contingencia-determinan el modo en que las tareas, los procesos, las unidades organizativas y la organización en su conjunto deberán ser diseñadas". Vemos, pues, que las aportaciones de la teoría contingente son importantes ya que por una parte ayudan a definir las dimensiones y variables de la organización y, por otra, vinculan las dimensiones organizativas o estructurales a los factores de contingencia de la empresa, siendo "la cuestión clave que plantea este enfoque (el contingente) ${ }^{10}$ la necesidad de que la empresa conceda una atención especial a los condicionantes que le imponen sus factores de contingencia" (Moreno-Luzón et al., 2001: 123).

\section{Definición de la teoría contingente estructural: Un aporte de Lex Donalson}

La teoría contingente estructural es un subconjunto del enfoque contingente (Donaldson, 1987, 2001). En ella, se argumenta que la estructura de la organización requiere estar ajustada a tres factores contextuales: el entorno, el tamaño y la estrategia, y de dicho ajuste depende en gran parte el desempeño y los resultados de la organización.

La teoría contingente estructural sostiene que los factores de contingencia tienen una influencia determinante en las características de la estructura organizativa los cuales pueden producir cambios y altos niveles de eficiencia en la organización -o en alguna parte de ella. En ese sentido, Donaldson, (2001: 6) sostiene que "en las estructuras mecánicas se produce una alta eficiencia cuando la contingencia de incertidumbre de tarea es baja, y en una estructura orgánica se produce una alta eficiencia cuando la contingencia de la incertidumbre de tarea es alta".

${ }^{10}$ Paréntesis nuestro. 
Del mismo modo, Donaldson $(1996,2001)$ sugiere que la teoría contingente estructural tiene tres elementos que conforman el centro de su paradigma:

1) Hay una asociación entre los factores de contingencia y la estructura de la organización;

2) Las contingencias determinan la estructura, si cambian esas contingencias cambia la estructura de la organización; y

3) Existe un nivel de ajuste necesario de las variables estructurales para cada nivel de contingencia que conduce a un alto nivel de desempeño, por consiguiente un desajuste lleva a un bajo desempeño.

En síntesis, hay un número de factores contextuales conectados a determinados aspectos de la estructura organizativa.

De igual forma, Donaldson (2001:6) aclara que el enfoque contingente estructural establece una asociación tri-variable entre el factor contingente y las variables de la estructura organizativa, esto quiere decir que el impacto de una variable sobre otra depende de una tercera variable. Así, "el efecto $X$ sobre $Y$ cuando $W$ es bajo, es diferente del efecto $X$ sobre $Y$ cuando $W$ es alto". Siendo la relación entre $X$ y $Y$, en parte, un sistema causal que implica la participación de una variable moderadora o condicionante. Esa situación la podemos observar en el modelo causal de la relación tamaño y estructura burocrática propuesto por el autor. A continuación se ofrece en la siguiente figura n. ${ }^{\circ} 3$. 
Figura N. ${ }^{\circ} 3$

Modelo causal de la relación tamaño-estructura burocrática

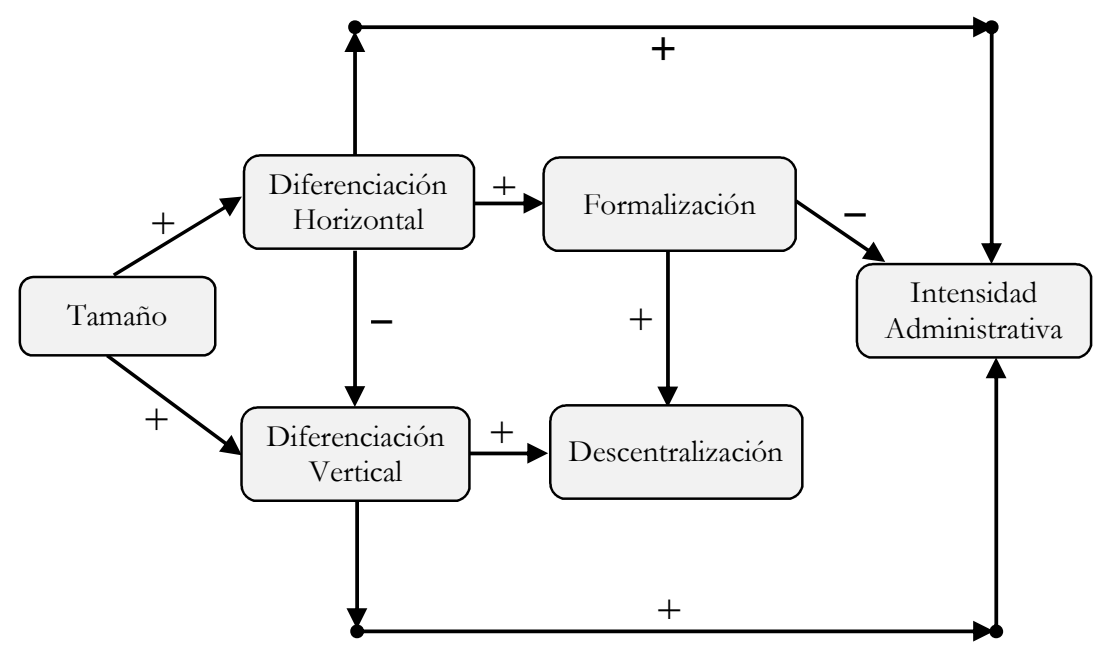

Fuente: Donaldson (1996, 2001).

Donaldson (1996) argumenta que la teoría contingente estructural es funcionalista y positivista. Funcionalista puesto que:

a) La estructura organizativa produce ciertos resultados funcionales asociados al objetivo de la organización;

b) La organización se mueve en el tiempo adoptando su estructura, de esa manera se ajusta a los niveles de contingencias que le permita alcanzar adecuados niveles de eficiencia; $y$

c) La estructura de la organización y sus cambios son el resultado de la adaptación funcional.

Por otra parte, la teoría contingente estructural es positivista puesto que:

a) Explica la estructura de la organización a través de elementos objetivos o materiales tales como el tamaño, la tecnología y el entorno en lugar de utilizar elementos subjetivos como las ideas, la ideología, la percepción y las normas; 
b) Está nutrida de investigaciones empíricas en lugar de la especulación; y

c) Aplica una metodología positivista en el sentido de que hay un extenso uso de la comparación científica entre investigaciones empíricas-cuantitativas.

\section{El significado del Fit en la teoría contingente}

De acuerdo a la teoría contingente, un alto desempeño en los resultados de la organización depende del fit o ajuste entre las variables de diseño organizativo y los factores del entorno. Así, la importancia de la teoría contingente se debe a su capacidad de entender y predecir en cierta forma el desempeño de la organización basado en el concepto del fit entre las variables organizacionales -incluyendo la estrategia, el tamaño, la estructura organizativa y la tecnología - y su entorno (Buttermann et al., 2008; Volberda, 2012). Lo que significa que dicho ajuste es una condición necesaria para que la organización pueda alcanzar los niveles de eficiencia y eficacia necesarios para mantenerse adecuadamente activa y competitiva, o de simplemente poder sobrevivir. Nadler y Tushman (1999) definen ese ajuste como la congruencia entre las contingencias, la estructura y estrategia.

El concepto del fit da respuesta al pensamiento general de la teoría de sistemas, en la idea de que los sistemas abiertos tienen una tendencia clara al equilibrio o auto organización, donde ponen en funcionamiento sus mecanismos de autocontrol y autorregulación que permiten ajustar sus componentes entre sí y con su entorno. El fit, en la organización como sistema abierto y en el marco de la teoría contingente, se reconoce en dos modelos básicos: " $\mathrm{La}$ bipótesis de congruencia, o necesaria adaptación entre la organización y los factores de contingencia; y la hipótesis de configuración que supone un ajuste, o fit, entre las variables organizativas"(Peris, 1995: 269). 
De igual forma, Mintzberg (1999: 219-220) indica que las estructuras efectivas requieren de una consistencia entre los parámetros de diseño ${ }^{11}$ y los factores de contingencia. La hipótesis de congruencia establece que una estructuración efectiva requiere que los parámetros de diseño se ajusten a los elementos de contingencia, y por tanto las organizaciones de éxito se diseñan en función a la situación que las rodea. Por su parte, la hipótesis de configuración plantea la existencia de un adecuado equilibrio interno y de ajuste entre las distintas variables o parámetros de diseño (Mintzberg, 1999; Nadler y Tushman, 1999).

Van de Ven y Drazin (1985) y Drazin y Van de Ven (1985) proponen tres enfoques conceptuales del fit, que emergen del desarrollo de la teoría contingente: selección, interacción y sistemas.

- Enfoque de selección. Asume que la base del ajuste, o fit, es la congruencia o relación entre el contexto de la organización y su estructura organizativa sin examinar si afecta o no el desempeño de la organización ${ }^{12}$. Se refiere a los estudios que plantean hipótesis en las cuales se muestra una fuerte correlación entre el contexto de la organización y su estructura organizativa, pero no discuten ni ofrecen evidencia alguna del efecto de esa congruencia sobre el desempeño o resultados.

- El enfoque de interacción. El ajuste o fit, se entiende aquí como una interacción entre el contexto y las variables de la estructura con un efecto importante sobre el desempeño de la organización. El centro del estudio bajo este enfoque no sólo es entender la congruencia, sino también explicar la variación del desempeño o los resultados a partir de esa interacción.

- El enfoque de sistemas. Considera al fit, como una consistencia interna entre las múltiples contingencias y características

11 Variables de diseño organizativo en otros autores.

12 Los autores consideran cuatro variables estructurales: centralización, formalización, diferenciación estructural y complejidad. 
estructurales que impactan en los resultados de la organización. Explican los autores, que los estudios que relacionan el concepto del fit con los enfoques de selección o interacción, tienden a centrarse sobre elementos contextuales únicos que afectan a las características estructurales únicas; es decir, "son enfoques reduccionistas que tratan las características de la organización como un cuerpo de elementos que pueden descomponerse para ser estudiados en forma independiente" (Drazin y Van de Ven, 1985:519). En este sentido, el enfoque de sistemas del fit hace énfasis en el análisis multivariable para examinar la consistencia entre las múltiples dimensiones del entorno, la estructura organizativa y el desempeño o resultados de la organización (Van de Ven y Drazin, 1985; Drazin y Van de Ven, 1985).

Por su parte, Miles y Snow (1984) dentro de la misma idea de Mintzberg (1999) y Nadler y Tushman (1999), argumentan que las organizaciones menos exitosas presentan pobres ajustes internos y externos, lo cual significa que gran parte del éxito de la empresa se debe al alcance del fit entre la estrategia, el entorno y un adecuado diseño de su estructura y procesos de gestión. Los autores explican cuatro tipos básicos de ajustes:

- Ajuste minimo. Aquel esencial que ocurre entre la estrategia, la estructura y los procesos necesarios para las operaciones básicas de la organización. En todo caso, se corresponde a un ajuste mínimo que permitirá su supervivencia en un entorno competitivo. Cualquier desajuste por largos períodos de tiempo ocasionará, casi con seguridad, el fracaso de la organización.

- Ajuste estricto. Para este enfoque, no basta con alcanzar el ajuste mínimo para lograr un verdadero desempeño con excelencia a mediano plazo, para ello es necesario una fuerte asociación entre la estrategia, la estructura y los procesos de gestión. Los autores sostienen que uno de los elementos condicionantes de un ajuste estricto es la presencia de una fuerte cultura organizativa. 
- Ajuste primario. Se vincula con la innovación o formulación de nuevas formas organizativas que conducen a una excelencia sustancial durante prolongados períodos de tiempo. Indican los autores que este ajuste implica un nuevo enfoque en la estrategia, en la estructura organizativa y en los procesos de gestión los cuales producen desempeños superiores, y, a su vez, le proporciona a la empresa un estatus diferenciador. Esta situación se relaciona con aquellas organizaciones que en un momento dado se convierten en modelos o paradigmas organizacionales.

- Ajuste frágil. Se da fundamentalmente cuando el entorno presenta altos niveles de dinamismo e inestabilidad que requieren cambios y ajustes de mucha importancia en la estrategia, la estructura y los procesos directivos. Pero sucede en ocasiones, que algunas empresas no son capaces de alcanzar los ajustes necesarios, por tanto disminuye su desempeño y aumenta el riesgo de fracasar, en consecuencia, a medio plazo pueden desaparecer.

Es importante destacar que en ciertas situaciones no sólo el entorno causa el deterioro del fit; también ocurren desajustes cuando la organización decide voluntariamente realizar cambios en su estrategia o en su estructura organizativa, debido a un interés particular por participar en nuevos mercados o incorporar innovaciones tecnológicas, productos o servicios (Miles y Snow, 1984). Se observa en el pensamiento de los autores la idea de que la participación y la acción de los gerentes también cuentan en el destino de la organización.

Por su parte, Donaldson $(1987,2001)$ dentro del marco de la teoría contingente-estructural, plantea que la actividad de ajuste se da de la siguiente manera:

1) La organización está inicialmente ajustada;

2) El nivel de ajuste de la organización se corresponde al nivel o exigencias de las variables contingentes; 
3) El fit o el estado de ajuste afecta positivamente el desempeño;

4) Cuando ocurren cambios en las contingencias, la organización retiene su estructura inicial por algún tiempo, por tanto su modificación o adaptación no es inmediata; y

5) Los cambios en las variables contingentes producen un desequilibrio y una disminución en el desempeño o eficiencia de la organización, presionándola a realizar los ajustes de sus variables internas, y por consiguiente la adopción de una nueva estructura organizativa.

En síntesis, los cambios en los factores de contingencia generan desajustes en las variables internas de la organización, siendo negativo su efecto con relación al fit. El desajuste produce un desempeño más bajo y cuando resulta menor a los niveles satisfactorios esperados por la empresa, ella procede a realizar los cambios adaptativos. Los mismos consisten en establecer, entre otras medidas, una nueva estructura organizativa o de incorporar ciertas adaptaciones en ella que se correspondan a los niveles de contingencias y así poder alcanzar nuevamente el adecuado fit que le permita mejorar su funcionamiento y resultados. Con lo cual, los cambios en la estructura responden a un efecto de retroalimentación negativo a partir del bajo desempeño producido, siendo este desajuste fruto de nuevas exigencias de los condicionantes contingentes.

\section{Conclusión}

Con el desarrollo del enfoque contingente se consideraron las oportunidades y las limitaciones ofrecidas por los factores de contingencia sobre el diseño de la organización y sus resultados. Esto, apoyándose en el hecho de que las variables organizativas tienen que conservar una estrecha relación o ajuste entre ellas y su entorno, de manera tal que la organización pueda alcanzar los niveles adecuados de funcionamiento para mantenerse efectivamente 
activa y obtener resultados superiores, o de simplemente para poder sobrevivir. Ese ajuste, o fit, condicionará la capacidad de la organización para competir y tener éxito. En resumen, el modelo contingente hace énfasis sobre el carácter determinista de un conjunto de factores contextuales como las causas principales de las características y el desempeño de la organización, dejando un papel subordinado a la actuación de sus directivos o gerentes.

Se entiende de la teoría contingente que el entorno y los subsistemas internos de cada organización, son de alguna manera únicos y sirven de base para diseñar y gestionar la organización de manera específica. De acuerdo a eso, ciertas organizaciones serán apropiadas para operaciones uniformes en un ambiente relativamente estable que permita un esquema de diseño organizativo bajo una jerarquía rígida, con un sistema de planificación y control muy preciso, y un alto grado de formalización. En contraste con otras organizaciones que operan en entornos inciertos y cambiantes, con un alto grado de innovación, las cuales podrían funcionar más eficientemente bajo un conjunto muy diferente de formas de gestión administrativa.

Cabe destacar y dentro de las ideas desarrolladas, que los cambios adaptativos son lentos, lo cual significa que la estructura organizativa se va ajustando progresivamente hasta alcanzar los niveles convenientes de equilibrio entre el conjunto de variables internas y los factores de contingencia. Es decir, que la influencia de esos factores no produce un efecto inmediato en la estructura organizativa, sus cambios se llevan a cabo cuando la organización, después de cierto tiempo, reacciona a los niveles de desempeño que considera no suficientemente satisfactorios.

Ahora bien, las reflexiones hechas en el presente trabajo y los fundamentos conceptuales desarrollados bajo una amplia revisión bibliografía, nos permiten plantear, con cierta seguridad, que la visión determinista de la teoría contingente, dentro del marco de la teoría de la organización, puede explicarse a través de un proceso de ajuste de los factores de contingencia: (a) con las variables de diseño 
organizativo; y (b) con la estructura organizativa necesaria para una organización y administración eficiente del trabajo que debe realizarse en la organización para obtener los bienes y servicio que demanda el entorno.

De esta manera, se plantea un modelo determinista-contingente de la organización a tres niveles de análisis organizativo:

1) Nivel micro-organizativo. Se ubican las variables de diseño organizativo, cuya definición y gestión dependerá básicamente de las características y naturaleza del trabajo requerido dentro de la organización para el diseño y elaboración de los bienes o servicios que demanda el entorno.

2) Nivel meso-organizativo. Se define la estructura organizativa básica necesaria tanto para una organización eficiente del trabajo, como para la gestión de las variables de diseño organizativo. Estas giran en torno a las estructuras: simples, funcional, divisional, matricial y en red.

3) Nivel macro-organizativo. Se configura la forma organizativa -orgánica, mecánica o burocrática- en función a la manera como se integren, gestionen y organicen los anteriores elementos que han sido influidos previamente, directa o indirectamente, por los factores de contingencia. Es decir, de la forma de administrar el trabajo, las variables de diseño y la estructura organizativa sabremos si la organización se está moviendo dentro del marco de los postulados de la teoría orgánica -forma orgánica o forma mecánica-, o de la teoría burocrática -forma burocrática en sus distintos grados de complejidad-

Las ideas anteriores se concretan a través del modelo que se presenta en la figura N. ${ }^{\circ} 4$. 


\section{Figura N. ${ }^{\circ} 4$ \\ Modelo Determinista-Contingente de la Organización}

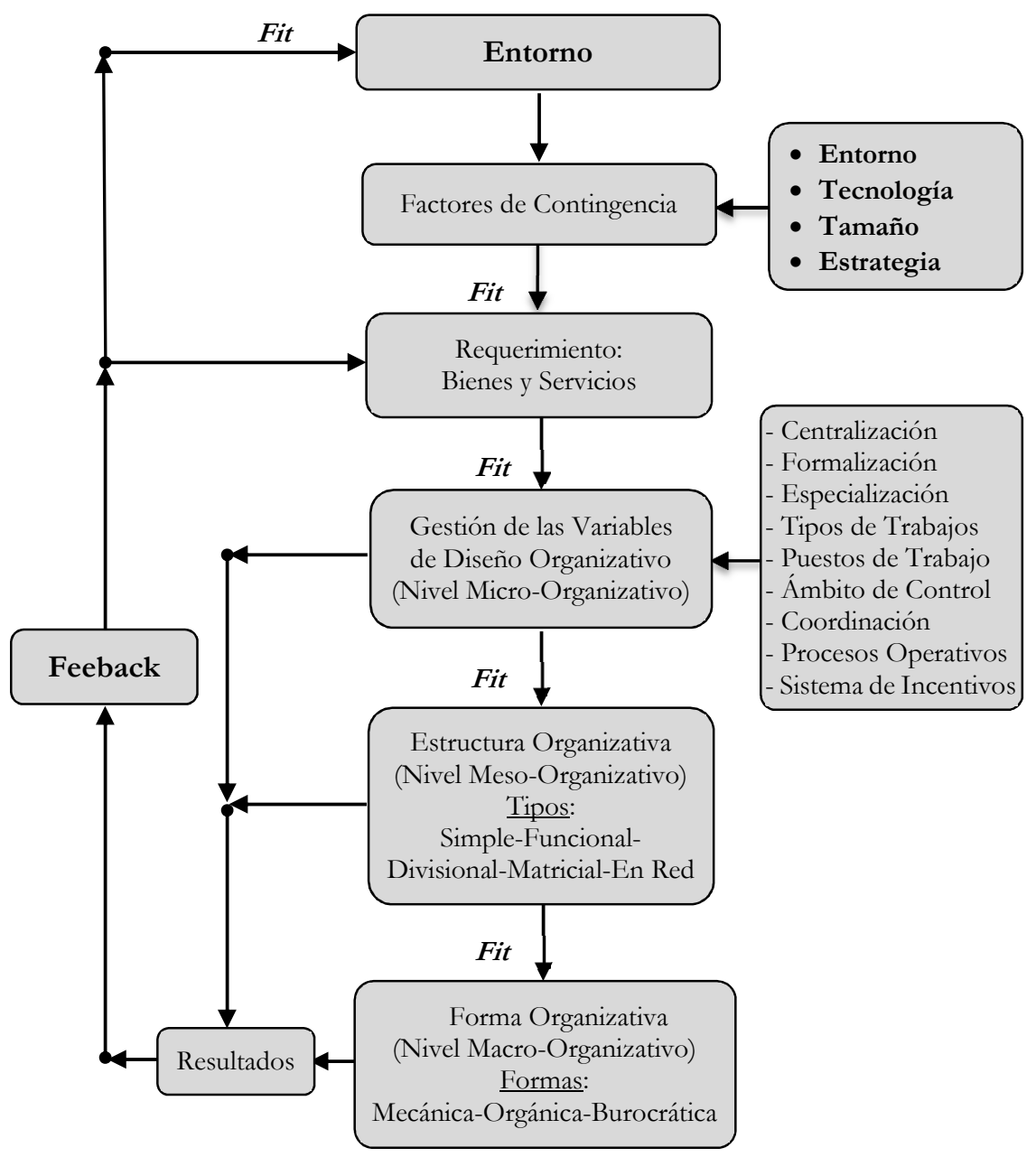

Fuente: Elaboración propia. 
El determinismo en la teoría contingente: Orígenes de sus fundamentos teóricos para su comprensión

\section{Referencias bibliográficas}

Astley, G., \& Van de Ven, A. (1983). Central perspective and debates in organizations theory. Administrative Science Quarterly, 28(2), 245-273.

Astley, G. (1985). Organizational size and bureaucratic structure. Organization Studies, 6(3), 201-228.

Blau, P. (1970). A formal theory of differentiation in organizations. American Sociological Review, 35(2), 201-218.

Burns, T., \& Stalker, G. M. (1961). The management of innovation. Londres: Tavistock Publications.

Buttermann, G., Germain, R., Iyer, K. (2008).Contingency theory "fit" as gestalt: An application to supply chain management. Transportation Research, Part E 4, 955-969.

Carvalho, C., Rossetto, C. R., \& Gonçalves, S. (2011). Determinismo e voluntarismo na adaptação estratégica: $\mathrm{O}$ caso de uma empresa de Varejo. Revista de Administração e Contabilidade da Unisinos, 8(4), 286-299.

Child, J. (1973). Predicting and understanding organization structure. Administrative Science Quarterly, 18(2), 168-185.

Child, J. L., \& Howard, D. (2003). The performance of cross-border units in China: A test of natural selection, strategic choice and contingency theories. Journal of International Business Studies, 43(3), 242-254.

Donaldson, L. (1987). Strategy and structural adjustment to regain fit and performance: In defense of contingency theory. Journal of Management Studies, 24(1), 1-24.

Donaldson, L. (1996). For positivist organization theory. California: Sage Publications. 
Donaldson, L. (2001). The contingency theory of organizations. California: Sage Publications.

Drazin, R., \& Van de Ven, A. (1985). Alternative forms of fit in contingency theory. Administrative Science Quarterly, 30(2), 514-539.

Fuente, J. de la, García-Tenorio, J., Guerra, L., \& Hernán Gómez, J. (2002). Diseño organizativo de la empresa. Madrid: Editorial Civitas.

Galbraith, J. (1973). Designing complex organizations. Massachusetts: Addison-Wesley.

Galbraith, J. (1977). Organization design. Massachusetts: AddisonWesley.

Lawrence, P., \& Lorsch, J. (1967a). Organization and environment. Managing differentiation and integration. Boston: Harvard University.

Lawrence, P., \& Lorsch, J. (1967b). Differentiation and integration in complex organizations. Administrative Science Quarterly, 12 (1), 1-47.

Mckinley, W. (1987). Complexity and administrative intensity: The case of declining organizations. Administrative Science Quarterly, 32, 87-105.

Miles, R., \& Snow, C. (1978). Organizational strategy, structure, and process. New York: McGraw-Hill.

Miles, R., \& Snow, C. (1984). Fit, failure and the hall of fame. California Management Review, 26(3), 0-28.

Mintzberg, H. (1999). The strategy process (3ra. Ed.). New Jersey: Prentice-Hall.

Moreno-Luzón, M., Peris, F., \& González, T. (2001). La gestión de la calidad y diseño de organizaciones: Teoría y estudio de casos. Madrid: Prentice Hall. 
El determinismo en la teoría contingente: Orígenes de sus fundamentos teóricos para su comprensión

Morgan, G. (1990). Imágenes de la organización. Madrid: Editorial Ra-Ma.

Nadler, D., \& Tushman, M. (1999). El diseño de la organización como arma competitiva. México: Ediciones Oxford.

Pertusa-Ortega, E., Molina-Azorín, J., \& Claver-Cortés, E. (2010). Competitive strategy, structure and firm performance. A comparison of the resource-based view and the contingency approach. Management Decision, 48(8), 1282-1303.

Peris, F. (1995). El soporte de la estrategia. Los enfoques de costes de transacción y de configuraciones-contingencias en el diseño de las organizaciones. En Á. Cuervo (Ed.). Dirección de empresas de los noventas., (pp. 253-277). Madrid: Civitas.

Pfeffer, J. \& Salancik, G. (1978). The external control of organizations: A resource dependence perspective. New York: Harper \& Row, Publishers.

Pugh, D. S., Hickson, D., J., Hinings, C. R., \& Turner C. (1968). Dimensions of organizational Structure. Administrative Science Quarterly, 13(1), 65-103.

Pugh, D. S., Hickson, D. J., \& Hinings, C. R. (1969a). An empirical taxonomy of structure of work organizations. Administrative Science Quarterly, 14(1), 115-126.

Pugh, D. S. M., Hickson, D. J., Hinings, C. R., \& Turner, C. (1969b). The context of organization structures. Administrative Science Quarterly, 14(1), 91-113.

Pugh, D. S. (1973). Does context determine form? Organizational dynamics. En D. S. Pugh Huff. (1997). Organization theory. (16-35). (4 ${ }^{\text {th }}$ Ed.). London: Penguin Books.

Pugh, D. S., \& Hickson, D. J. (1976). Organizational structure in its context: The Aston Programmed I. Michigan: Saxon House Studies. 
Pugh, D. S (1981). The Aston program perspective. En: A. Van de Ven y J. William (Eds.). Perspectives on organization design and behavior. (135-166). New York: John Wiley \& Sons.

Rond, M. D., \& Thietart, R-A. (2007). Choice, chance, and inevitability in strategy. Strategic Management Journal, 28, 535-551.

Sillince, J. (2005). A contingency theory of rhetorical congruence, Academy of Management Review, 30(3), 608-621.

Simon, C. (2007). A configuration dorm of fit in management accounting contingency Theory. An empirical investigation. The Business Review, 7(2), 220-227.

Tenhiälä, A. (2011). Contingency theory of capacity planning: The link between process types and planning methods. Journal of Operations Management, 29, 65-77.

Thompson, J. (1967). Organización en acción. México: McGraw-Hill Interamericana, S. A.

Van de Ven, A., \& Drazin, R. (1985). The concept of fit in contingency theory. Research in Organizational Behavior, 7, 333-365.

Volberda, H., Niels Van Der, W., Verwaal, E., Stienstra, M., \& Verdu, A. (2012). Contingency fit, institutional fit, and firm performance: A meta fit approach to organization-environment relationships. Organization Science, 23(4), 1040-1054.

Wong, C. Y., Boon-itt, S., \& Wong, C. (2011). The contingency effects of environmental uncertainty on the relationship between supply chain integration and operational performance. Journal of Operations Management, 29, 604-615.

Zapata-Rotundo, G., \& Caldera, J. (2008). La complejidad de las organizaciones: Aproximación a un modelo teórico. Revista de Ciencias Sociales, 14(1), 46-62. 
Zapata-Rotundo, G., Mirabal, A., \& Hernández, A. (2009). Modelo teórico conceptual de la estructura organizativa: Un análisis contingente. Ciencia y Sociedad, 34(4), 618-640.

Zapata-Rotundo, G., \& Hernández, A. (2011). La empresa: Diseño, estructura y formas organizativas. Barquisimeto: Editorial Horizonte, C. A.

Zapata-Rotundo, G. J., \& Mirabal, A. (2013). Contingencia, voluntarismo y strategic choice: Un análisis teórico de sus enfoques sobre el entorno y el diseño de la organización. Universidad y empresa, 15(34), 119-139. 


\section{Dr. Gerardo Zapata Rotundo}

Analista de Sistemas (UCLA, 1985), licenciado en Contaduría Pública (UCLA, 1993); especialista en Auditoría (UCLA, 1998) y doctor en Dirección de Empresas por la Universidad de Valencia, España, (2006). Docente-investigador Titular del Decanato de Administración y Contaduría (DAC) de la Universidad Centroccidental "Lisandro Alvarado" (UCLA), Venezuela. Posee experiencia docente en el área de informática, organización y métodos, sistemas de información y sistemas administrativos. Miembro del Comité Editor de la revista científica Compendium (DAC/UCLA). Su línea de investigación se concentra en organización de empresas y teoría de las organizaciones, con diversas publicaciones en revistas científicas, libros y ponencias en congresos.

Email: zapager@yahoo.com

Recibido: 05/01/2014 Aprobado: 11/02/2014 